\title{
A Wiener-Type Condition for Boundary Continuity of Quasi-Minima of Variational Integrals
}

\author{
Emmanuele DiBenedetto* \\ Department of Mathematics, Vanderbilt University \\ 1326 Stevenson Center, Nashville TN 37240, USA \\ email: em.diben@vanderbilt.edu \\ Ugo Gianazza \\ Dipartimento di Matematica "F. Casorati", Università di Pavia \\ via Ferrata 1, 27100 Pavia, Italy \\ email: gianazza@imati.cnr.it
}

\begin{abstract}
A Wiener-type condition for the continuity at the boundary points of Q-minima, is established, in terms of the divergence of a suitable Wiener integral [1.8) and Theorem 1.1].
\end{abstract}

AMS Subject Classification (2010): Primary 49K20, 35J25; Secondary 35B45

Key Words: Wiener criterion, Continuity, Capacity, DeGiorgi classes, Quasi-Minima

\section{Introduction}

Let $E$ be a bounded, open subset of $\mathbb{R}^{N}$ and let $f: E \times \mathbb{R}^{N+1} \rightarrow \mathbb{R}$ be a Carathéodory function satisfying

$$
C_{o}|D u|^{p} \leq f(x, u, D u) \leq C_{1}|D u|^{p},
$$

for constants $0<C_{o} \leq C_{1}$, and some fixed $p>1$. A function $u \in W_{\operatorname{loc}}^{1, p}\left(\mathbb{R}^{N}\right)$ is a Q-sub(super)minimum for the functional

$$
J(u)=\int_{E} f(x, u, D u) d x
$$

*Supported by NSF grant DMS-1265548 
if there exists $Q \geq 1$ such that

$$
J(u) \leq Q J(u-(+) \varphi),
$$

for all non-negative functions $\varphi \in W_{\text {loc }}^{1, p}\left(\mathbb{R}^{N}\right)$ with $\operatorname{supp} \varphi \subset \bar{E}$. A function $u \in W_{\text {loc }}^{1, p}\left(\mathbb{R}^{N}\right)$ is a Q-minimum for $J$ if it satisfies (1.3) for all $\varphi \in W_{\text {loc }}^{1, p}\left(\mathbb{R}^{N}\right)$ with $\operatorname{supp} \varphi \subset \bar{E}$ and no further sign restriction ([5]). A Q-minimum $u$ takes boundary values $g \in W_{\text {loc }}^{1, p}\left(\mathbb{R}^{N}\right)$ on $\partial E$ if

$$
u-g \in W_{o}^{1, p}(E) .
$$

If $g \in C\left(\mathbb{R}^{N}\right)$ one asks under what conditions on $\partial E$, the boundary datum $g$ is taken by $u$ in the sense of continuous functions. Let $y \in \partial E$, denote with $B_{\rho}(y)$ the ball of radius $\rho$ about $y$. For $1<p<N$, the $p$-capacity of the compact set $E^{c} \cap \bar{B}_{\rho}(y)$ is defined by

$$
c_{p}\left[E^{c} \cap \bar{B}_{\rho}(y)\right]=\inf _{\substack{\psi \in W_{o}^{1, p}\left(\mathbb{R}^{N}\right) \cap C\left(\mathbb{R}^{N}\right) \\ E^{c} \cap \bar{B}_{\rho}(y) \subset[\psi \geq 1]}} \int_{\mathbb{R}^{N}}|D \psi|^{p} d x .
$$

For $1<p<N$, the relative $p$-capacity of $E^{c} \cap \bar{B}_{\rho}(y)$ with respect to $B_{\rho}(y)$ is

$$
\delta_{y}(\rho)=\frac{c_{p}\left[E^{c} \cap \bar{B}_{\rho}(y)\right]}{\rho^{N-p}}, \quad(1<p<N) .
$$

If $p=N$, and for $0<\rho<1$, the $N$-capacity of the compact set $E^{c} \cap \bar{B}_{\rho}(y)$, with respect to the ball $B_{2}(y)$, is defined by

$$
c_{N}\left[E^{c} \cap \bar{B}_{\rho}(y)\right]=\inf _{\substack{\psi \in W_{o}^{1, N}\left(B_{2}(y)\right) \cap C_{o}\left(B_{2}(y)\right) \\ E^{c} \cap \bar{B}_{\rho}(y) \subset[\psi \geq 1]}} \int_{B_{2}(y)}|D \psi|^{N} d x .
$$

The relative capacity $\delta_{y}(\rho)$ can be formally defined by (1.6), for all $1<p \leq N$ : for $p=N, \delta_{y}(\rho) \equiv c_{N}\left[E^{c} \cap \bar{B}_{\rho}(y)\right]$, as defined by (1.7).

For a positive parameter $\epsilon$ denote by $I_{p, \epsilon}(y, \rho)$ the Wiener integral of $\partial E$ at $y \in \partial E$, i.e.,

$$
I_{p, \epsilon}(y, \rho)=\int_{\rho}^{1}\left[\delta_{y}(t)\right]^{\frac{1}{\epsilon}} \frac{d t}{t} .
$$

The main result of this note is:

Theorem 1.1 Let $u$ be a $Q$-minimum for the functional $J(u)$, for $1<p \leq N$. Assume that $u$ takes a continuous datum $u=g$ on $\partial E$ in the sense of (1.4). There exists $\epsilon \in(0,1)$, and $\gamma>1$, that can be determined apriori, quantitatively only in terms of $N, p, Q$, and the ellipticity ratio $\frac{C_{1}}{C_{o}}$, such that for all $y \in \partial E$, and all $\rho \in(0,1)$

$$
\underset{E \cap B_{\rho}(y)}{\operatorname{ess} \operatorname{osc}} u \leq \gamma \max \left\{\underset{\partial E \cap B_{\rho}(y)}{\operatorname{osc}} g ;\left(\underset{E \cap B_{1}(y)}{\operatorname{Oos}} u\right) \exp \left(-I_{p, \epsilon}(y, \rho)\right)\right\} .
$$

Thus, when $1<p \leq N$, a Q-minimum $u$, when given continuous boundary data $g$ on $\partial E$, is continuos up to $y \in \partial E$, if the Wiener integral $I_{p, \epsilon}(y, \rho)$ diverges as $\rho \rightarrow 0$. If $p>N$ the continuity of $u$, is insured by the Sobolev embedding theorem. 


\subsection{Novelty and Significance}

The celebrated Wiener criterion states that a harmonic function in $E$ is continuous up to $y \in \partial E$ if and only if the Wiener integral $I_{2,1}(y, \rho)$ diverges as $\rho \rightarrow 0$ ([10]). Next, for a given $g \in W^{1, p}\left(\mathbb{R}^{N}\right) \cap C\left(\mathbb{R}^{N}\right)$ consider the boundary value problem

$$
\begin{array}{ll}
u-g \in W_{o}^{1, p}(E), & \text { for } p>1, \\
\operatorname{div} \mathbf{a}(x, u, D u)=0, & \text { weakly in } E,
\end{array}
$$

where, the vector field $\mathbf{a}$ is subject to the structure conditions

$$
\begin{aligned}
\mathbf{a}(x, u, D u) \cdot D u & \geq C_{o}|D u|^{p}, \\
|\mathbf{a}(x, u, D u)| & \leq C_{1}|D u|^{p-1}
\end{aligned}
$$

for constants $0<C_{o} \leq C_{1}$, and some fixed $p>1$. The prototype is

$$
\begin{array}{ll}
u-g \in W_{o}^{1, p}(E) & \text { for } p>1, \\
\operatorname{div}\left(|D u|^{p-2} D u\right)=0 & \text { weakly in } E .
\end{array}
$$

For solutions of (1.12) Theorem 1.1 is due to Maz'ja ([7]), with the optimal value of the parameter $\epsilon=(p-1)$. The proof is based on the comparison principle and the Harnack inequality. For solutions of (1.10)-11.11) the result is due to Gariepy and Ziemer (4]), still for optimal value of the parameter $\epsilon=(p-1)$. For these quasi-linear equations there is not, in general, a maximum principle. Their proof is based on the Moser's logarithmic estimates ([8]) leading to the Harnack inequality for some proper convex functions of the solutions, near the boundary point $y \in \partial E$. In their approach, the structure of the p.d.e. in (1.10)-(1.11) is crucial.

Each such quasi-linear equation is the Euler equation of a functional $J$, for a suitable integrand $f(x, u, D u)([5)$. The notion of Q-minimum is considerably more general as it includes almost minimisers, or even minimisers of functionals $J(u)$ which do not admit a Euler equation due to the possible lack of Gateaux differentiability of $J$.

Nevertheless Q-minima share several crucial properties of solutions of quasilinear equations of the type (1.10)-(1.11). For example, they are locally bounded and locally Hölder continuous in $E$. Their interior continuity carries at those boundary points where $\partial E$ has positive geometric density ([5]). Moreover, nonnegative Q-minima satisfy the Harnack inequality ([3). However, Q-minima are not known to satisfy a maximum principle, nor Harnack inequalities near $\partial E$.

The significance of a Wiener condition for Q-minima, is that the structure of $\partial E$ near a boundary point $y \in \partial E$, for $u$ to be continuous up to $y$, hinges on minimizing a functional, rather than solving an elliptic p.d.e.

The only result, to date, in this direction, states that a Q-minimum $u$, with continuous boundary data $g \in C(\partial E)$, is continuous up to a boundary point $y \in \partial E$ if $([11])$

$$
\int_{\rho}^{1} \exp \left(-\frac{1}{\delta_{y}(t)^{\frac{1}{p-1}}}\right) \frac{d t}{t} \rightarrow \infty \quad \text { as } \rho \rightarrow 0 .
$$


Ziemer's proof follows from a standard DeGiorgi iteration technique ([2]). The novelty of our Theorem 1.1 is in replacing the exponential decay (1.13) in the Wiener integral with a power-like decay. The technical novelty is in extending a weak Harnack inequality for quasi minima ([3]), to hold near the boundary, coupled with proper choices of test functions in (1.3) as indicated by Tolksdorf ([9]). The optimal value of the parameter $\epsilon=(p-1)$, remains elusive.

Acknowledgement. We are grateful to the anonymous referee for the valuable comments. After submitting this note, we learnt that a similar result has been proved with a different technique by J. Björn (see [1]).

\section{Main Tools in the Proof of Theorem 1.1}

\subsection{Q-Subminima and Test Functions}

Proposition 2.1 Let $y \in \partial E$ and let $u$ be a non-negative $Q-$ subminimum for $J$, in $\bar{B}_{\rho}(y) \cap \bar{E}$, such that $u=0$ on $B_{\rho}(y) \cap \partial E$. There is a positive constant $\gamma_{o}$ that can be determined apriori only in terms of $N, p, Q$, and the ellipticity ratio $\frac{C_{1}}{C_{o}}$, such that

$$
\int_{B_{\rho}(y) \cap E}|D u|^{p}|\varphi|^{p} d x \leq \gamma_{o} \int_{B_{\rho}(y) \cap E} u^{p}|D \varphi|^{p} d x
$$

for all non-negative $\varphi \in W_{o}^{1, p}\left(B_{\rho}(y)\right)$.

Note that $\varphi$ is not required to vanish on $B_{\rho}(y) \cap \partial E$. The proof results from a minor variant of an argument of Tolksdorf [9]. From the property (1.1) of $f$ and the definition (1.2)-(1.3) of Q-subminimum,

$$
\int_{B_{\rho}(y) \cap E}|D u|^{p} d x \leq Q \frac{C_{1}}{C_{o}} \int_{B_{\rho}(y) \cap E}|D(u-u \varphi)|^{p} d x
$$

for all non-negative $\varphi \in W_{o}^{1, p}\left(B_{\rho}(y)\right)$. The new observation here is that since $u$ vanishes on $B_{\rho}(y) \cap \partial E$, the test function $u \varphi$ is admissible in (1.3) even if $\varphi$ does not vanish on $B_{\rho}(y) \cap \partial E$, provided it does vanish on $\partial B_{\rho}(y)$. The remaining arguments leading to (2.1) starting from (2.2) are identical to those in 9 .

Corollary 2.1 Let $u$ satisfy the same assumptions as Proposition 2.1. Then for all constants $h>0$

$$
\int_{B_{\rho}(y) \cap E}|D(u+h)|^{p}|\varphi|^{p} d x \leq \gamma_{o} \int_{B_{\rho}(y) \cap E}(u+h)^{p}|D \varphi|^{p} d x
$$

for all non-negative $\varphi \in W_{o}^{1, p}\left(B_{\rho}(y)\right)$. The constant $\gamma_{o}$ is the same as in 2.1) and is independent of $h$. 


\section{$2.2 \quad Q$-Superminima and the Weak Harnack Inequality}

Proposition 2.2 Let $y \in \partial E$ and let $v \in W^{1, p}\left(B_{2 \rho}(y)\right)$ be non-negative and satisfying

$$
\int_{B_{r}(z)}\left|D(v-k)_{-}\right|^{p} d x \leq \frac{\gamma_{1}}{r^{p}} \int_{B_{2 r(z)}}(v-k)_{-}^{p} d x
$$

for all balls $B_{2 r}(z) \subset B_{2 \rho}(y)$ and all $k>0$, for a constant $\gamma_{1}$ independent of $k, z$ and $r$. Then, there exist constants $C>1$ and $\epsilon \in(0,1)$, that can be determined apriori only in terms of $N, p$, and the constant $\gamma_{1}$ in 2.4), such that

$$
\left(\frac{1}{\left|B_{\rho}(y)\right|} \int_{B_{\rho}(y)} v^{\epsilon} d x\right)^{\frac{1}{\epsilon}} \leq C \underset{B_{\rho}(y)}{\operatorname{essinf}} v .
$$

The weak Harnack inequality (2.5) is a sole consequence of the family of inequalities (2.4), and as such, disconnected from the notion of Q-superminimum ([3]). However, if $v$ is a Q-superminimum in $E$, for balls $B_{2 \rho}(y) \subset E$, inequalities (2.4) are satisfied by $v([5])$.

\section{Proof of Theorem 1.1}

\subsection{Estimating the Oscillation About a Point $y \in \partial E$ by the Weak Harnack Inequality}

Having fixed $y \in \partial E$ assume without loss of generality that $y=0$ and write $B_{\rho}(0)=B_{\rho}$, and continue to denote by $g$ the boundary datum of $u$, in the sense of (1.4). We may assume that at least one of the following two inequalities holds true:

$$
\begin{aligned}
& \underset{B_{2 \rho} \cap E}{\operatorname{ess} \sup } u-\frac{1}{4} \underset{B_{2 \rho} \cap E}{\operatorname{ess} \operatorname{osc}} u>\underset{B_{2 \rho} \cap \partial E}{\operatorname{ess} \sup } g ; \\
& \underset{B_{2 \rho} \cap E}{\operatorname{ess} \inf } u+\frac{1}{4} \underset{B_{2 \rho} \cap E}{\operatorname{ess} \operatorname{osc}} u<\underset{B_{2 \rho} \cap \partial E}{\operatorname{essinf}} g .
\end{aligned}
$$

Indeed, if both are violated one has

$$
\underset{B_{2 \rho} \cap E}{\operatorname{ess} \operatorname{osc}} u \leq 2 \underset{B_{2 \rho} \cap \partial E}{\operatorname{ess} \text { Osc }} g,
$$

and the assertion of the theorem follows. Assuming then that the first holds, the function

$$
\left(u-\left(\underset{B_{2 \rho} \cap E}{\operatorname{ess} \sup } u-\frac{1}{4} \underset{B_{2 \rho} \cap E}{\operatorname{ess} \operatorname{Osc}} u\right)-(1-k) \frac{1}{4} \underset{B_{2 \rho} \cap E}{\operatorname{ess} \operatorname{osc}} u\right)_{+}
$$

is a non-negative Q-subminimum, for $J$, in $\bar{B}_{2 \rho} \cap \bar{E}$, for all $0<k \leq 1$, vanishing on $B_{2 \rho} \cap \partial E$. As such it satisfies (2.1) of Proposition 2.1] over $B_{2 \rho}$, which we 
rewrite as

$$
\begin{aligned}
& \int_{B_{2 \rho} \cap E} \mid\left.D(w-(1-k))_{+}\left|{ }^{p}\right| \varphi\right|^{p} d x \\
& \leq \gamma_{o} \int_{B_{2 \rho} \cap E}(w-(1-k))_{+}^{p}|D \varphi|^{p} d x
\end{aligned}
$$

for all non-negative $\varphi \in W_{o}^{1, p}\left(B_{2 \rho}\right)$, where

$$
w \stackrel{\text { def }}{=} \frac{\left(u-\left(\underset{B_{2 \rho} \cap E}{\operatorname{ess} \sup } u-\frac{1}{4} \underset{B_{2 \rho} \cap E}{\operatorname{ess} \operatorname{osc}} u\right)\right)}{\frac{1}{4} \underset{B_{2 \rho} \cap E}{\operatorname{esc} \operatorname{Osc} u}},
$$

for all $0<k \leq 1$. From the definitions one verifies that $0 \leq w \leq 1$, and it vanishes on $B_{2 \rho} \cap \partial E$. We continue to denote by $w$ and $(w-(1-k))_{+}$their extensions with zero on $B_{2 \rho} \cap E^{c}$. By Corollary 2.1, inequalities (3.1) continue to hold for all $k \geq 0$. Set $v=1-w$ and rewrite (3.1) in the form

$$
\int_{B_{2 \rho}}\left|D(v-k)_{-}\right|^{p}|\varphi|^{p} d x \leq \gamma_{o} \int_{B_{2 \rho}}(v-k)_{-}^{p}|D \varphi|^{p} d x
$$

for all non-negative $\varphi \in W_{o}^{1, p}\left(B_{2 \rho}\right)$, and for all $k \geq 0$. In what follows we denote by $\gamma$ a generic, positive constant that can be quantitatively determined apriori only in terms of $N, p, Q$.

For a ball $B_{2 r}(z) \subset B_{2 \rho}$, in (3.2) choose $\varphi$ as the standard, non-negative cutoff function in $B_{2 r}(z)$ which equals 1 on $B_{r}(z)$ and such that $|D \varphi| \leq r^{-1}$.

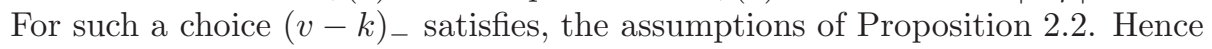
there exists $\gamma>1$ and $\epsilon \in(0,1)$ that can be determined apriori only in terms of $N, p, Q$, and the ellipticity ratio $\frac{C_{1}}{C_{o}}$, such that

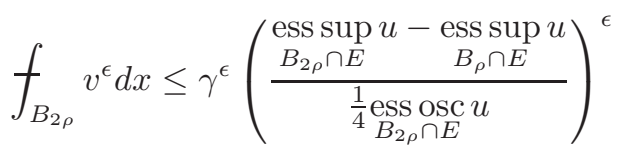

Remark 3.1 Whence the parameter $\epsilon$ has been identified, inequality (3.3) continues to hold for smaller $\epsilon$, with the same constant $\gamma$.

\subsection{Estimating the Oscillation About a Point $y \in \partial E$ by the Capacity of $E^{c} \cap \bar{B}_{\rho}(y)$}

Continue to assume $y=0$ and write $B_{\rho}(0)=B_{\rho}$.

Proposition 3.1 There exists $p_{o} \in(1, p)$, that depends only on $N, p, Q$, and the ellipticity ratio $\frac{C_{1}}{C_{o}}$, such that for all $p_{o} \leq q<p$, and for all non-negative $\zeta \in W_{o}^{1, p}\left(B_{2 \rho}\right)$, there holds

$$
\int_{B_{2 \rho}} v^{-q}|D v|^{p} \zeta^{p} d x \leq \gamma \int_{B_{2 \rho}} v^{p-q}|D \zeta|^{p} d x
$$


for a constant $\gamma>1$ that depends only on $N, p, Q, q, p_{o}$, and the ellipticity ratio $\frac{C_{1}}{C_{o}}$.

Proof: Using an idea of [6], set $\varphi=v^{\sigma} \zeta$ in (3.2) where $\sigma \in(0,1)$ is a parameter to be chosen and $\zeta \in W_{o}^{1, p}\left(B_{2 \rho}\right)$ is non-negative. For such choices (3.2) yields

$$
\int_{B_{2 \rho}}\left|D(v-k)_{-}\right|{ }^{p} v^{\sigma p} \zeta^{p} d x \leq \gamma \int_{B_{2 \rho}}(v-k)_{-}^{p}\left[\sigma^{p} v^{(\sigma-1) p}|D v|^{p} \zeta^{p}+v^{\sigma p}|D \zeta|^{p}\right] d x .
$$

Choose $\sigma>0$ and $1<q<p$ so that $(1-\sigma) p<q$, multiply both sides of this inequality by $k^{-\sigma p-q-1}$ and integrate in $d k$ over $(0, \infty)$. Interchanging the order of integration with the aid of Fubini's theorem, the left-hand side equals

$$
\int_{0}^{\infty} \int_{B_{2 \rho}}\left|D(v-k)_{-}\right|{ }^{p} v^{\sigma p} \zeta^{p} k^{-\sigma p-q-1} d x d k=\frac{1}{\sigma p+q} \int_{B_{2 \rho}}|D v|^{p} v^{-q} \zeta^{p} d x .
$$

The right-hand side is transformed and estimated by

$$
\begin{aligned}
\int_{0}^{\infty} & \int_{B_{2 \rho}}(v-k)_{-}^{p}\left[\sigma^{p} v^{(\sigma-1) p}|D v|^{p} \zeta^{p}+v^{\sigma p}|D \zeta|^{p}\right] k^{-\sigma p-q-1} d x d k \\
& =\sigma^{p} \int_{B_{2 \rho}} v^{(\sigma-1) p}|D v|^{p} \zeta^{p}\left(\int_{v}^{\infty} k^{(1-\sigma) p-q-1} d k\right) d x \\
& +\int_{B_{2 \rho}} v^{\sigma p}|D \zeta|^{p}\left(\int_{v}^{\infty} k^{(1-\sigma) p-q-1} d k\right) d x \\
& =\frac{1}{q-(1-\sigma) p} \int_{B_{2 \rho}} v^{(1-\sigma) p-q}\left[\sigma^{p} v^{-(1-\sigma) p}|D v|^{p} \zeta^{p}+v^{\sigma p}|D \zeta|^{p}\right] d x .
\end{aligned}
$$

Combining these estimates yields

$$
\begin{aligned}
\int_{B_{2 \rho}} v^{-q}|D v|^{p} \zeta^{p} d x \leq & \gamma \frac{\sigma p+q}{q-(1-\sigma) p} \sigma^{p} \int_{B_{2 \rho}} v^{-q}|D v|^{p} \zeta^{p} \\
& +\gamma \frac{\sigma p+q}{q-(1-\sigma) p} \int_{B_{2 \rho}} v^{p-q}|D \zeta|^{p} d x .
\end{aligned}
$$

To conclude the proof choose $\sigma \in(0,1)$ such that

$$
\gamma \frac{\sigma p+q}{q-(1-\sigma) p} \sigma^{p}=\frac{1}{2}, \quad \text { and } \quad(1-\sigma) p<q<p .
$$

One may first choose $p_{o}=\left(1-\sigma^{2}\right) p \leq q<p$ and then $\sigma$ so small that the first of these inequalities is in force.

We now conclude the proof of the Theorem, still following [6]. Fix $p_{o} \leq q<p$ where $p_{o}$ is the parameter claimed in Proposition 3.1 and rewrite it as $q=p-\epsilon$. By virtue of Remark 3.1 this value of $\epsilon$ can be taken equal to the analogous in (3.3). For such a choice, (3.4) gives

$$
\int_{B_{2 \rho}}\left|D\left[v^{\frac{\epsilon}{p}} \varphi\right]\right|^{p} d x \leq \gamma(\epsilon) \int_{B_{2 \rho}} v^{\epsilon}|D \varphi|^{p} d x
$$


Next choose $\varphi \in W_{o}^{1, p}\left(B_{2 \rho}\right)$ to be the standard, non-negative cutoff function in $B_{2 \rho}$ which equals 1 on $B_{\rho}$ and such that $|D \varphi| \leq \rho^{-1}$. For such a choice and $\rho$ sufficiently small $v \varphi=1$ on $B_{\rho} \cap E^{c}$ and therefore,

$$
c_{p}\left[E^{c} \cap \bar{B}_{\rho}\right] \leq \frac{\gamma(\epsilon)}{\rho^{p}} \int_{B_{2 \rho}} v^{\epsilon} d x .
$$

Dividing by $\rho^{N-p}$ and combining the resulting inequality with (3.3) gives

$$
\delta_{o}^{\frac{1}{\epsilon}}(\rho) \leq \gamma \frac{\underset{B_{2 \rho} \cap E}{\operatorname{ess} \sup } u-\underset{B_{\rho} \cap E}{\operatorname{ess} \sup } u}{\frac{1}{4}_{B_{2 \rho} \cap E}^{\operatorname{ess} \operatorname{Osc} u}} .
$$

This in turn implies

$$
\underset{B_{\rho} \cap E}{\operatorname{ess} \operatorname{Osc}} u \leq\left(1-\frac{1}{4 \gamma} \delta_{o}(\rho)\right) \underset{B_{2 \rho}}{\operatorname{ess} \operatorname{osc} u}
$$

Iteration of this inequality over a sequence of balls of dyadic radii $\rho_{-n}=2^{-n} \rho$ yields the Theorem.

\section{References}

[1] J. Björn, Sharp exponents and a Wiener type condition for boundary regularity of quasiminimizers, Preprint, 2015, 1-13.

[2] E. DeGiorgi, Sulla differenziabilità e l'analiticità degli integrali multipli regolari, Mem. Accad. Sci. Torino Cl. Sci. Fis. Mat. Natur. (3), t. 3, (1957) 25-43.

[3] E. DiBenedetto and N.S. Trudinger, Harnack inequalities for quasi-minima of variational integrals, Ann. Inst. Henri Poincaré, Analyse Non Linéaire, 1(4), (1984), 295-308.

[4] R. Gariepy and W.P. Ziemer, Behavior at the boundary of solutions of quasilinear elliptic equations, Arch. Rational Mech. Anal., 56 (1974/75), 372-384.

[5] M. Giaquinta and E. Giusti, Quasi-Minima, Ann. Inst. Henri Poincaré, Analyse Non Linéaire, 1, (1984), 79-10\%.

[6] J.H. Michael and W.P. Ziemer, Interior regularity for solutions to obstacle problems, Nonlinear Anal., 10, (1986), 12, 1427-1448.

[7] V. Maz'ja, On the continuity at a boundary point of solutions of quasi-linear elliptic equations, Vestnik Leningrad Univ. Math. 3 (1976), 225-242.

[8] J. Moser, A new proof of de Giorgi's theorem concerning the regularity problem for elliptic differential equations, Comm. Pure Appl. Math., 13, (1960), 457-468. 
[9] P. Tolksdorf, Remarks on Quasi(sub)Minima, Nonlinear Anal. 10(2), (1986), 115-120.

[10] N. Wiener, Une condition nécessaire et suffisante de possibilité pour le problème de Dirichlet Comptes Rendus, Acad. de Sci. Paris, 178, (1924), $1050-1054$.

[11] W.P. Ziemer, Boundary Regularity for Quasiminima, Arch. Rational Mech. Anal. 92 (1986), 4, 371-382. 